Wright State University

CORE Scholar

Mechanical and Materials Engineering Faculty

Publications

Mechanical and Materials Engineering

3-1-2016

\title{
Carbon-Based Hierarchical Scaffolds for Myoblast Differentiation: Synergy between Nano-Functionalization and Alignment
}

\author{
Akhil Patel \\ Shilpaa Mukundan \\ Wenhu Wang \\ Wright State University - Main Campus \\ Anil K. Karumuri \\ Vinayak Sant
}

See next page for additional authors

Follow this and additional works at: https://corescholar.libraries.wright.edu/mme

Part of the Materials Science and Engineering Commons, and the Mechanical Engineering Commons

\section{Repository Citation}

Patel, A., Mukundan, S., Wang, W., Karumuri, A. K., Sant, V., Mukhopadhyay, S. M., \& Sant, S. (2016).

Carbon-Based Hierarchical Scaffolds for Myoblast Differentiation: Synergy between Nano-

Functionalization and Alignment. Acta Biomaterialia, 32, 77-88.

https://corescholar.libraries.wright.edu/mme/256

This Article is brought to you for free and open access by the Mechanical and Materials Engineering at CORE Scholar. It has been accepted for inclusion in Mechanical and Materials Engineering Faculty Publications by an authorized administrator of CORE Scholar. For more information, please contact library-corescholar@wright.edu. 


\section{Authors}

Akhil Patel, Shilpaa Mukundan, Wenhu Wang, Anil K. Karumuri, Vinayak Sant, Sharmila M. Mukhopadhyay, and Shilpa Sant

This article is available at CORE Scholar: https://corescholar.libraries.wright.edu/mme/256 
Full length article

\title{
Carbon-based hierarchical scaffolds for myoblast differentiation: Synergy between nano-functionalization and alignment
}

\author{
Akhil Patel $^{\mathrm{a}}$, Shilpaa Mukundan ${ }^{\mathrm{a}}$, Wenhu Wang ${ }^{\mathrm{b}}$, Anil Karumuri ${ }^{\mathrm{b}}$, Vinayak Sant ${ }^{\mathrm{a}}$, \\ Sharmila M. Mukhopadhyay ${ }^{\mathrm{b}}$, Shilpa Sant ${ }^{\mathrm{a}, \mathrm{c}, \mathrm{d}, * *}$ \\ a Department of Pharmaceutical Sciences, School of Pharmacy, University of Pittsburgh, Pittsburgh, PA 15261, United States \\ ${ }^{\mathrm{b}}$ Center for Nanoscale Multifunctional Materials, Mechanical E' Materials Engineering, Wright State University, Dayton, OH 45324, United States \\ ${ }^{\mathrm{c}}$ Department of Bioengineering, Swanson School of Engineering, University of Pittsburgh, Pittsburgh, PA 15261, United States \\ ${ }^{\mathrm{d}}$ McGowan Institute for Regenerative Medicine, University of Pittsburgh, Pittsburgh, PA 15219, United States
}

\section{A R T I C L E I N F O}

\section{Article history:}

Received 14 September 2015

Received in revised form 4 December 2015

Accepted 5 January 2016

Available online $\mathrm{xxxx}$

\section{Keywords:}

Carbon nanotubes

Multiscale hierarchy

Myogenesis

Carbon-based scaffolds

Alignment

Interconnected microporous structure

$\mathrm{C} 2 \mathrm{C} 12$

Nano-roughness

\begin{abstract}
A B S T R A C T
While several scaffolds have been proposed for skeletal muscle regeneration, multiscale hierarchical scaffolds with the complexity of extracellular matrix (ECM) haven't been engineered successfully. By precise control over nano- and microscale features, comprehensive understanding of the effect of multiple factors on skeletal muscle regeneration can be derived. In this study, we engineered carbon-based scaffolds with hierarchical nano- and microscale architecture with controlled physico-chemical properties. More specifically, we built multiscale hierarchy by growing carbon nanotube (CNT) carpets on two types of scaffolds, namely, interconnected microporous carbon foams and aligned carbon fiber mats. Nanostructured CNT carpets offered fine control over nano-roughness and wettability facilitating myoblast adhesion, growth and differentiation into myocytes. However, microporous foam architecture failed to promote their fusion into multinucleated myotubes. On the other hand, aligned fibrous architecture stimulated formation of multinucleated myotubes. Most importantly, nanostructured CNT carpets interfaced with microscale aligned fibrous architecture significantly enhanced myocyte fusion into multinucleated mature myotubes highlighting synergy between nanoscale surface features and micro-/macroscale aligned fibrous architecture in the process of myogenesis.
\end{abstract}

\section{Statement of Significance}

Due to limited regenerative potential of skeletal muscle, strategies stimulating regeneration of functional muscles are important. These strategies are aimed at promoting differentiation of progenitor cells (myoblasts) into multinucleated myotubes, a key initial step in functional muscle regeneration. Recent tissue engineering approaches utilize various scaffolds ranging from decellularized matrices to aligned biomaterial scaffolds. Although, majority of them have focused on nano- or microscale organization, a systematic approach to build the multiscale hierarchy into these scaffolds is lacking. Here, we engineered multiscale hierarchy into carbon-based materials and demonstrated that the nanoscale features govern the differentiation of individual myoblasts into myocytes whereas microscale alignment cues orchestrate fusion of multiple myocytes into multinucleated myotubes underlining the importance of multiscale hierarchy in enhancing coordinated tissue regeneration.

(c) 2016 Published by Elsevier Ltd. on behalf of Acta Materialia Inc.

\section{Introduction}

According to US Bureau of Labor statistics, about 100,000 cases of muscle injuries are reported in year 2011 [1]. Similarly, injuries

* Corresponding author at: 3501, Terrace Street, 527 Salk Hall, Pittsburgh, PA 15261, United States.

E-mail address: shs149@pitt.edu (S. Sant). due to trauma and tumor ablation procedures result in skeletal muscle damage. Due to limited regenerative potential of the muscle, therapeutic strategies aimed at functional muscle regeneration are gaining importance. Some of the strategies used in the past such as transplant of autologous muscle and injection of satellite cells have shown limited success due to donor site morbidity, poor integration and survival of implanted tissue or cells [2]. These problems can be circumvented by designing the regenerative 
strategies that aim to recreate the microenvironment conducive for myogenesis.

Multiscale hierarchical extracellular matrix (ECM) architecture at the macro-, micro- and nanoscale along with biomimetic cellECM interactions are some of the most important features to be considered for the development of functional muscle tissue which itself possesses multiscale hierarchy [3,4]. Cell-interactive ECM-mimicking materials can facilitate essential cellular processes for normal muscle growth, development and regeneration $[3,5,6]$. Specifically, a combination of biochemical and biophysical stimuli are critical for guiding appropriate orientation of progenitor cells to promote formation of aligned and well-organized muscle bundles [7]. These biophysical stimuli include nano-roughness, topographical guidance and electrically conductive surface. Surface topography, especially nanoscale surface roughness can be an important feature in improving cell-scaffold interaction and cell adhesion [8]. Since skeletal muscle tissue is electrically excitable, electroactive materials allow local delivery of electrical stimulus to enhance cell proliferation, differentiation and tissue regeneration. Indeed, conductive materials have shown to improve myoblast differentiation into myocytes $[9,10]$. Surface wettability is another factor that affects cell adhesion on the scaffold surface. For example, moderately water-wettable surfaces with contact angles around $70^{\circ}$ facilitate better attachment of cells to the scaffolds through strong binding of cellular adhesion proteins [11].

Apart from nanoscale features, many native tissues including muscles display multiscale hierarchical structure responsible for their unique architecture and function [3]. Submicron scale myofibrils constitute the basic structural units, which further organize into muscle fibers (10-80 microns in diameter), which then bundle together to form muscle fasciculus and eventually, a functional muscle [12]. Thus, to enhance regeneration of a functional skeletal muscle, it is desirable to recreate multiscale hierarchical scaffolds that are composed of repeating small structural units to create a three-dimensional architecture. In addition, macro- and microscale scaffold architecture provides contact guidance to the myoblast cells. This includes different microscale features such as interconnected pores [13] or aligned fibrous structure [14]. Similarly, macro-porous architecture of the scaffold is essential for easy exchange of nutrients and waste products as well as facilitating cell infiltration throughout the scaffold volume $[13,15]$. Honeycomb-like polymer foams have been investigated for cardiac muscle regeneration and peculiar interconnected honey-comb architecture was thought to reinforce the scaffold during the continuous contraction and relaxation process of myocardial tissue $[16,17]$. Thus, for functional skeletal muscle regeneration, it is essential to build multiscale hierarchical scaffolds that provide contact guidance to myoblasts through nano-roughness, provide alignment cues at microscale and allow cell infiltration as well as nutrient exchange.

Scaffolds possessing each of these individual features have been built but combining multiple such features into a single scaffold and comprehensive understanding of their interplay in generation of a functional muscle tissue is yet to be explored. This is especially a daunting task given that all the materials cannot be processed into controlled hierarchical architecture with simultaneous control over their physico-chemical properties. Electrically conductive carbon-based scaffolds can be processed to possess multiscale hierarchy along with macro-porous structure and different geometries. They have been widely used as conductive materials in energy storage-related applications [18]. Recently, carbon-based materials are gaining popularity in various tissue engineering applications such as osteogenesis [19], neural cell growth [20,21], bone implants [22] and drug delivery [23]. For example, Li et al. reported that three-dimensional macro-porous graphene foams provided electrically conductive and macro-porous surface and promoted proliferation and differentiation of neural stem cells [21].

In this study, we engineered multiscale hierarchy into carbonbased materials with controlled nanoscale (nano-roughness, wettability) and microscale features (highly interconnected pores vs highly aligned fibers). More specifically, we processed carbon materials into two types of multiscale hierarchical scaffolds; namely, interconnected porous carbon foams [24] and aligned carbon fiber mats. The $200-500 \mu \mathrm{m}$ size micro-pores with wall width of $50-80 \mu \mathrm{m}$ were structural repeating units in reticulated carbon foams while carbon microfibers (diameter 5-6 $\mu \mathrm{m}$ ) were repeating units in aligned carbon fiber mats. We further introduced nanoscale features by growing tailored carpet-like arrays of carbon nanotubes (CNTs) on the micro-pore walls of foams and surfaces of fibers. Such surface functionalization offers nanoscale roughness, increased specific surface area and tailored physicochemical properties like wettability. In this study, we specifically investigated the integrated effects of multiscale hierarchy, that is, microscale features (interconnected pores vs. aligned fibers) and surface nanofunctionalization (CNT/Si-CNT) on differentiation of mouse myoblasts into multinucleated myotubes. It was hypothesized that the CNT nano-functionalization of carbon-based materials will facilitate biomimetic cell-material interaction and promote myoblast differentiation, while the highly ordered fiber alignment will enhance fusion of differentiated myocytes into multinucleated myotubes.

\section{Materials and methods}

\subsection{Materials}

Cell culture media, Dulbecco's phosphate buffered saline (DPBS) and serum were purchased from Mediatech Inc. (Manassas, USA) or Corning Inc. (NY, USA) unless otherwise specified. The C2C12 cells were a kind gift from Dr. Feinberg at Carnegie Mellon University, Pittsburgh, PA, USA. CNTs used in this study possess average length of $17 \mu \mathrm{m}$ and a diameter of $18 \mathrm{~nm}$. It consists of 1\% (Atomic\%) Fe.

\subsection{Nano-functionalization of carbon foams and fibers}

Reticulated vitreous carbon (RVC) foams are typically fabricated from the pyrolysis of thermosetting polymer, resulting in vitreous carbon struts. RVC foams selected in this study were with 80 pores per inch (PPI), provided by Ultramet Inc. Carbon nanotube carpets were synthesized using a two-step process previously developed by Mukhopadhyay group [25,26]. The first step involves deposition of silicon oxide activation/buffer layer via room temperature Microwave Plasma Enhanced Chemical Vapor Deposition reactor (MPE-CVD). The hexamethyldisiloxane [HMDSO, Sigma Aldrich, 99.5\%] was used as a precursor in conjunction with 99.9\% pure oxygen. This is followed by chemical vapor deposition of CNT using xylene and ferrocene [Alfa Aesar, 99\%] as a carbon and catalyst source, respectively. Silica ( $\mathrm{SiOx}$ ) coating was applied using methods published previously [24]. Briefly, acid catalyzed sol-gel process was utilized in order to produce the silica coating on the CNT-grafted hierarchical substrate using Tetraethyl orthosilicate (TEOS, Sigma-Aldrich; reagent grade, 98\%) as a precursor. Plain weave carbon fiber mats were obtained from Hexcel (ACGP206P) and functionalized with CNTs as described above for the foams.

\subsection{Measurement of contact angle}

The contact angles of pristine and functionalized foams and fibers were measured using a dynamic contact angle goniometer 
set-up in the Mukhopadhyay laboratory. Details of this wellestablished experimental set-up are described in an earlier paper [24]. A drop of de-ionized water was added on the samples using a $21 \mathrm{G}$ needle and the image was captured after $10 \mathrm{~s}$. The images were processed and contact angle was measured using SolidWorks.

\subsection{Scanning electron microscopy (SEM)}

\subsubsection{Imaging}

Surface characterization of as-prepared and cell-seeded Foam and Fiber scaffolds was carried out using SEM (JEOL 9335 Field Emission SEM) [27]. Cell-seeded scaffolds were fixed following 21 days and 14 days of culture for Foams and Fibers, respectively. SEM was performed following air drying for $24 \mathrm{~h}$. Scaffolds were sputter-coated with $5 \mathrm{~nm}$ of gold-palladium using Cressington 108 auto sputter coater. Images were obtained using accelerated voltage of $3 \mathrm{kV}$ and a working distance of $8 \mathrm{~mm}$.

\subsubsection{Elemental Analysis using energy dispersive spectroscopy (EDS)}

Elemental composition was determined using energy dispersive spectroscopy (EDS) at $7 \mathrm{Kv}$ operating voltage. When the solid to be analyzed is exposed to a beam of electrons in the Scanning Electron Microscope, the atoms in the sample emit secondary, backscattered X-rays, whose energies are characteristic of the atomic element. By analyzing the peaks of X-ray energies using EDS detector, elements present in the sample are identified.

\subsection{C2C12 mouse myoblast culture}

The mouse myoblast cells (C2C12) cells were cultured in Dulbecco's modified Eagle's medium (DMEM) supplemented with $10 \%$ of heat inactivated Fetal Bovine Serum (HyClone ${ }^{\mathrm{TM}}$ ) and $1 \%$ antibiotic (Penicillin and streptomycin). The cells were cultured in T75 flasks in a humidified incubator $\left(37^{\circ} \mathrm{C}\right.$ and $\left.5 \% \mathrm{CO} 2\right)$, media was changed every 2 days and cells were split $1: 3$ at $70 \%$ confluence.

\subsection{Cell seeding on foams and fibers}

The cylindrical foams of height $0.5 \mathrm{~cm}$ and diameter $0.5 \mathrm{~cm}$ were sterilized for $30 \mathrm{~min}$ in $70 \%$ isopropanol under UV. The foam scaffolds were seeded with a seeding density of $1 \times 10^{6}$ cells/scaffold. For metabolic activity measurement, the cells were seeded and cultured for 14 days in growth medium. For differentiation studies, the scaffolds were cultured in growth media until day 3 followed by additional 18 days in differentiation media (DMEM supplemented with $10 \%$ heat inactivated horse serum, HyClone ${ }^{\mathrm{TM}}$ ). Fiber mats of size $1 \mathrm{~cm} \times 1 \mathrm{~cm}$ were seeded with C2C12 cells at a seeding density of 90,000 cells/mat (normalized based on the volume of mats). The study was carried out over a period of 14 days.

\subsection{Metabolic activity of cells}

The metabolic activity of cells seeded on Foams or Fibers $(n=3)$ were measured using the alamarBlue ${ }^{\circledR}$ assay (Thermo Scientific, USA). The assay was performed over a period of 14 days. alamarBlue ${ }^{\circledR}$ solution $(10 \% \mathrm{v} / \mathrm{v})$ was prepared in complete growth media and $1 \mathrm{~mL}$ of this solution was added to each well containing cell-seeded scaffold and incubated for $4 \mathrm{~h}$ at $37^{\circ} \mathrm{C}$. After this step, $100 \mu \mathrm{L}$ of the solution from each well was transferred to 96-well plate and the fluorescence intensity was measured at excitation/ emission wavelength of 530/590 nm using the microplate reader (Synergy HT, BioTek instruments). The wells containing only alamarBlue $^{\circledR}$ solution in media was used for background fluorescence correction. Pristine-Foams and Pristine-Fibers were used as control to compare the proliferation on functionalized Foams and Fibers.

\subsection{Immunofluorescence staining}

Cell-seeded Foams or Fibers were fixed with 4\% paraformaldehyde for $30 \mathrm{~min}$ and washed 3 times with DPBS followed by the blocking/ permeabilization with DPBS containing $0.1 \%$ Triton $\mathrm{X}-100$ and 5\% BSA for $1 \mathrm{~h}$ at room temperature. The scaffolds were then incubated with the primary antibody against myosin heavy chain (MHC), MF-20 (1:50, DSHB, Iowa) overnight at $4{ }^{\circ} \mathrm{C}$ and washed with DPBS three times. The scaffolds were then stained with secondary antibody (Alexa Fluor 488-conjugated goat antimouse IgG, 1:1000, Santa Cruz Biotechnology Inc., USA) for 1 hour at room temperature followed by three times washing with DPBS. The cell nuclei were stained with Hoechst 33342 (Thermo Fisher, USA).

\subsection{Confocal microscopy}

Confocal images were obtained using inverted confocal laser scanning microscope (Olympus Fluoview 1000). Lasers of 488-, $559-$, and 633-nm wavelength were used. Objective lenses of $20 \mathrm{X}$ and $40 \mathrm{X}$ were used to acquire the z-stack images with $5 \mu \mathrm{m}$ thickness of each $z$ slice. Data is presented as maximum intensity projection of the z-stack.

\subsection{Image analysis}

NIH Image software was used for quantification of confocal images. Percentage MHC-positive cells were calculated by taking a ratio of MHC-positive cells to the total number of cells in at least 3 images per scaffold type. At least 120 total nuclei were considered per scaffold type. Myotube fusion index was calculated by taking a ratio of number of fused cells containing 2 or more nuclei to total number of nuclei per image for the Fiber scaffolds. Number of nuclei per myotube was measured manually. Myotube maturation index was measured from the immunofluorescence images by calculating ratio of myotubes with 5 or more nuclei to the total number of myotubes. The graphs are plotted using GraphPad Prism 6 and Origin Pro 2015.

\subsection{Statistical analysis}

The data are represented as mean \pm standard deviation ( $n=4-5)$. The statistical significance between the groups was analyzed using one-way or two-way ANOVA for multiple comparisons followed by Tukey post-hoc analysis (GraphPad Prism 6). p values less than 0.05 were considered statistically significant.

\section{Results and discussion}

3.1. Nano-functionalization of foam scaffolds affects nano-roughness and wettability

The carbon-based scaffolds with interconnected porous structure were obtained from Ultramet Inc. They are fabricated from pyrolysis of thermosetting polymer, resulting in vitreous carbon struts as described previously [24,25]. Surface functionalization with nanoscale features is known to alter wettability and nanoroughness [24], which, in turn, may affect cell adhesion, spreading and differentiation. In this study, CNTs are chosen for surface functionalization as they have been shown to promote myoblast differentiation $[9,28,29]$. Along with electrical conductivity, CNTs also impart nano-roughness [24] which may be beneficial for cell adhesion and spreading. Indeed, when encapsulated in gelatin methacrylate hydrogels, CNTs have promoted spreading and elongation of cardiomyocytes [30]. Hence, multiscale hierarchical 
carbon foams with interconnected microporous structure were nano-functionalized with carpets of CNT to modulate nanoroughness and wettability. CNT carpets were synthesized using previously reported two-step process $[25,26]$; first step involves the coating of silica nanolayer and the second step involves growth of CNT carpets by chemical vapor deposition (CVD). This process ensures growth of immobilized CNT carpets on the surface of the scaffolds as shown in earlier papers [24,25]. Non-functionalized foam scaffolds and CNT functionalized foam scaffolds are hereafter referred as Pristine-Foam and CNT-Foam, respectively (schematic in Fig. 1A). Detailed multiscale architecture of nanofunctionalized foams is depicted in Supplementary Fig. 1.

The wettability of Pristine-Foam and CNT-Foam was measured by contact angle of water droplet on the scaffold surface. Surface nano-functionalization with CNT is known to increase the contact angle of carbon foam surfaces [24]. Indeed, CNT grafting on the foam surface increased the contact angle from $65-75^{\circ}$ to $160^{\circ}$ (Table 1 ), making the surfaces less wettable. Similar increase in contact angle and decrease in surface wetting was also reported for poly-L-lactic acid surfaces coated with multiwall CNTs [8]. Such decrease in wettability of CNT-coated poly-L-lactic acid surfaces was attributed to nano-roughness.

Nano-roughness of CNT-Foam was characterized using scanning electron microscopy (SEM). Fig. 1B shows SEM images of Pristine-Foam and CNT-Foam. As shown in Figs. 1B1 and B3, Pristine-Foam and CNT-Foam exhibited interconnected porous structure with overall similar pore size/porosity [25]. CNT carpet of about $20 \mu \mathrm{m}$ thickness was found to grow perpendicular to the surface of Pristine-Foam scaffolds (Fig. 1B4, white arrows). Successful CNT functionalization was further confirmed by high magnification $(20,000 \mathrm{X})$ images of the walls of functionalized foam showing uniform fibrous carpet of CNTs rendering nanoscale roughness to these surfaces (Fig. 1B5) compared to smooth surface observed for Pristine-Foam scaffolds (Fig. 1B2). Thus, CNT functionalization increased the contact angle rendering the surface less wettable and also increased the nano-roughness of CNT-Foams. These results indicate that CNT functionalization affected multiple factors, such as surface wettability and nano-roughness that would further influence the cell-scaffold interaction. Indeed, less wettable surfaces tend to promote the adsorption of proteins such as laminin and fibronectin, which further disrupt ordered water molecule, leading to increased entropy. Such entropy-driven adsorption is usually strong and irreversible compared to enthalpy-driven and reversible adsorption on easily wettable surfaces [31,32]. Protein adsorption further plays important role in cell adhesion. On the other hand, nano-roughness has shown to influence cell attachment and proliferation by facilitating better cell-material and cell-cell interaction $[8,33,34]$.

CNT-Foams were further functionalized with amorphous silica (SiOx) (referred as Si-CNT-Foam hereafter) layer. Silica is a hydrophilic, inorganic, bio-resorbable and biocompatible material that has been widely investigated in tissue engineering, specifically in bone regeneration [35]. Silica functionalization on CNT will change the wettability, as demonstrated in an earlier study [24], while maintaining the nano-roughness same as that of CNT-Foam. This will decouple the effect of wettability and nano-roughness on cell adhesion/spreading. Successful nano-functionalization of CNTs with Si was confirmed by the elemental analysis of Si-CNTFoams, which showed increase in the percentage of Si atoms from $1.38 \%$ (CNT-Foam) to $4.32 \%$ (Si-CNT-Foam) (Table 2). It could also be seen that the water contact angle of the CNT carpets decreased from $160.3^{\circ}$ (CNT-Foam) to $66.5^{\circ}$ (Si-CNT-Foam) (Table 1 ) implying that Si-CNT-Foam is no longer hydrophobic despite the presence of CNT carpet morphology. We characterized nano-roughness of SiCNT-Foam using SEM. As shown in Fig. 1B6, Si-CNT-Foam exhibited interconnected porous structure with similar pore size as Pristine
Foam and CNT-Foam. Si-CNT coating was found to be perpendicular to the surface of the scaffold (Fig. 1B7, white arrow) similar to that of CNT-Foam (Fig. 1B4). As shown in Fig. 1B8, uniform fibrous carpet of Si-CNT conferred nano-roughness to the surface, similar to CNT-Foam (Fig. 1B5). In conclusion, Si-CNT-Foam possesses comparable macro-porosity, contact angle and wettability to that of Pristine-Foam with added nano-roughness of CNT-Foam.

Following the characterization of the Foam scaffolds, we assessed their cytocompatibility as well as ability to promote cell adhesion, spreading, and differentiation.

\subsection{Effect of nano-roughness and wettability on myoblast adhesion, proliferation and differentiation into multinucleated myotubes}

Although CNTs have been utilized in many biomedical applications [28-30], their cytocompatibility still remains a concern. Cytotoxic effects of CNTs have been studied extensively [36]. CNTs were found to be cytotoxic when they were freely suspended in culture and were available for cellular uptake [37]. We hypothesized that immobilization of CNT carpets on Pristine-Foams through strong interaction with underlying silica substrate nanolayer (Supplementary Fig. 1) will prevent shedding of individual CNTs and minimize their cellular uptake. In our previous study on the physico-chemical characterization of Pristine and CNT-Foams $[25,26]$, we have shown that interaction between CNT and underlying silica nanolayer is much stronger than that between graphitic layers of the Pristine-Foam.

We, therefore, tested the hypothesis that immobilized CNTs/Si-CNTs will exhibit excellent cytocompatibility and promote myoblast growth and differentiation into myocytes by virtue of structural cues such as, nano-roughness, wettability, conductive surfaces and macro-porous scaffold architecture. We cultured mouse myoblast (C2C12) cells on pristine and functionalized foam scaffolds (Fig. 2A). Infiltration of $\mathrm{C} 2 \mathrm{C} 12$ cells into the scaffolds was studied after 21 days of culture using SEM. Fig. 2B1, B2 and B3 indicated that cells were able to infiltrate and adhere (white arrows) uniformly to all the foam scaffolds. In case of Pristine-Foams (Fig. 2B1 and Supplementary Fig. 2a), cells adhered to the foam wall, forming a uniform cell sheet over the surface, whereas the presence of nano-hair like CNTs in the other two scaffolds increased surface roughness and cell-scaffold interaction in the functionalized scaffolds as evident from the cells (pseudo colored in red, black asterisks) attached on to these hair-like projections (Supplementary Fig. 2b, black arrows). Thus, SEM images suggest that nano-functionalization with CNT carpets enhanced cellFoam interactions in the functionalized foam scaffolds. De novo matrix synthesis on biomaterial scaffolds is one of the essential steps in tissue regeneration. Production of functional tissuemimetic structures can be facilitated through the synthesis of new ECM by the seeded cells along with biodegradation of the scaffolds. In a study reported by Sant et al., Collagen type 1, fibronectin and laminin were secreted by valvular interstitial cells on poly (glycerol sebacate)-poly ( $\varepsilon$-caprolactone) nanofibrous scaffolds [38]. In the present study, Pristine-Foam, CNT-Foam and Si-CNTFoam facilitated de novo ECM secretion on the surface of scaffolds as shown by white arrows in Supplementary Fig. 3. This data suggest that all three foam scaffolds are bioactive and facilitate cell adhesion, spreading and de novo matrix secretion.

The effect of surface nano-roughness and wettability on cell adhesion and proliferation of $\mathrm{C} 2 \mathrm{C} 12$ was further investigated by determining their viability with alamarBlue ${ }^{\circledR}$ assay (Fig. 2C). AlamarBlue $^{\circledR}$ assay measures ability of metabolically active cells to reduce resazurin to fluorescent resorufin [27]. All scaffolds supported growth of $\mathrm{C} 2 \mathrm{C} 12$ as evident from the significant increase in their metabolic activity over a period of 7 days. It is noteworthy that differences between Pristine-Foams and CNT/Si-CNT-Foam 


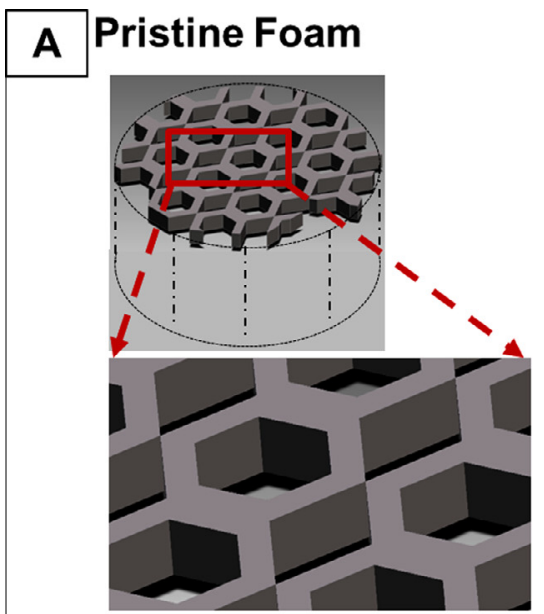

\section{CNT-Foam}
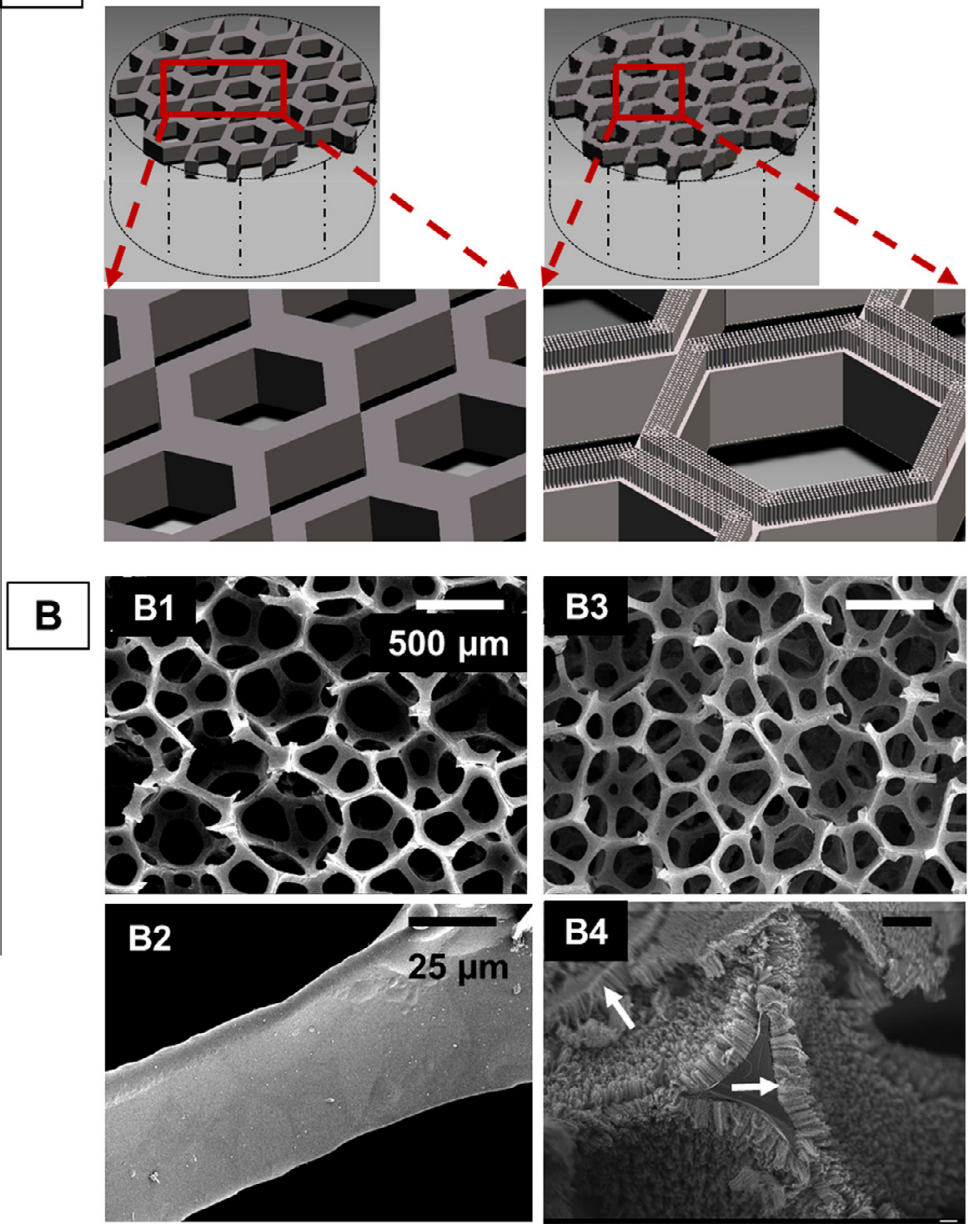

Table 1

\begin{tabular}{|c|c|}
\hline Composition & $\begin{array}{c}\text { Contact } \\
\text { angle }\left(^{\circ}\right)\end{array}$ \\
\hline Pristine-Foam & $65-75^{\circ}$ \\
\hline CNT-Foam & $160^{\circ}$ \\
\hline Si-CNT Foam & $66.5^{\circ}$ \\
\hline
\end{tabular}
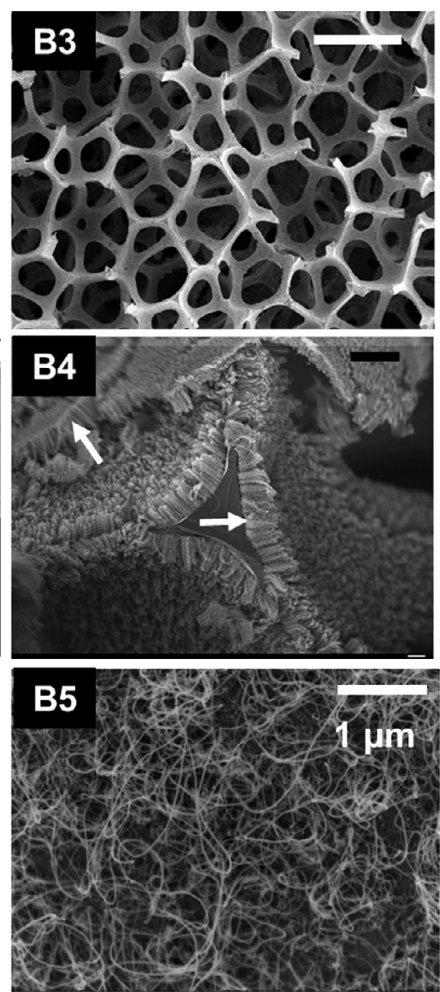

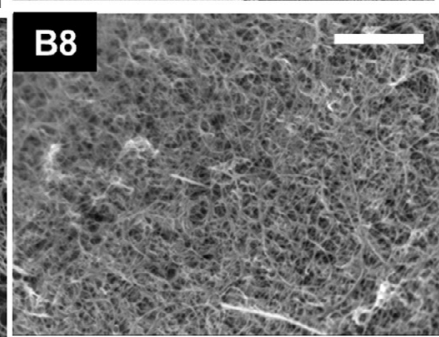

Table 2

\begin{tabular}{|c|c|c|c|}
\hline Element & $\begin{array}{c}\text { Pristine- } \\
\text { Foam } \\
\text { (At } \%)\end{array}$ & $\begin{array}{c}\text { CNT-Foam } \\
\text { (At\%) }\end{array}$ & $\begin{array}{c}\text { Si-CNT- } \\
\text { Foam } \\
\text { (At\%) }\end{array}$ \\
\hline $\mathrm{C}$ & 91.81 & 95.62 & 86.79 \\
\hline $\mathrm{O}$ & 08.19 & 02.81 & 07.81 \\
\hline $\mathrm{Si}$ & ND & 01.38 & 04.32 \\
\hline $\mathrm{Fe}$ & ND & 00.19 & 01.08 \\
\hline
\end{tabular}

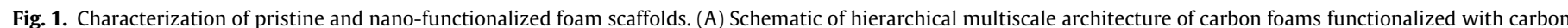

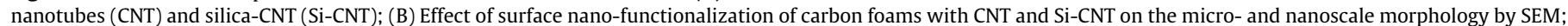

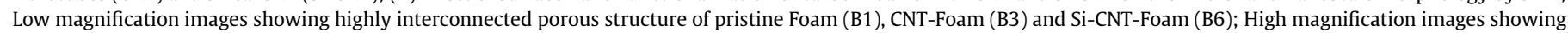

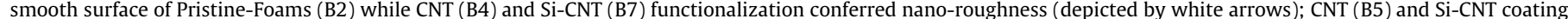

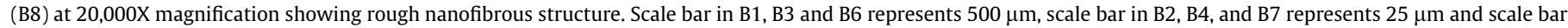

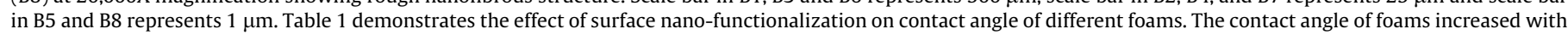

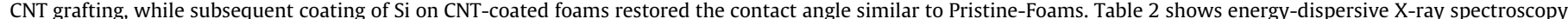

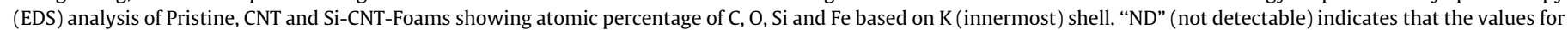

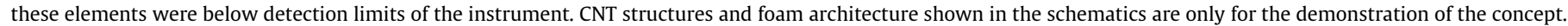
Schematics not to scale.

Please cite this article in press as: A. Patel et al., Carbon-based hierarchical scaffolds for myoblast differentiation: Synergy between nano-functionalization and alignment, Acta Biomater. (2016), http://dx.doi.org/10.1016/j.actbio.2016.01.004 

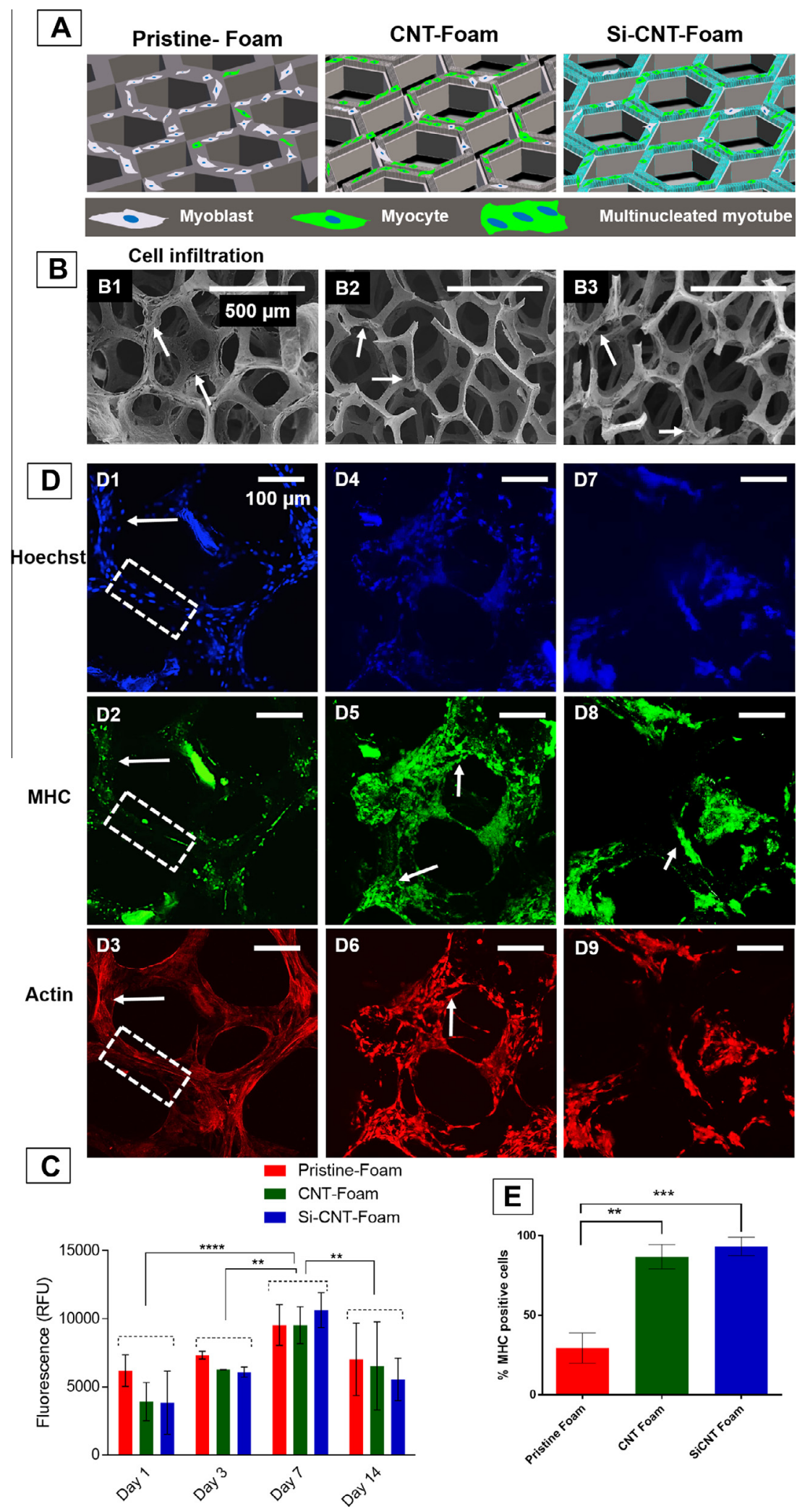

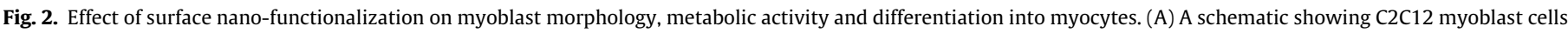

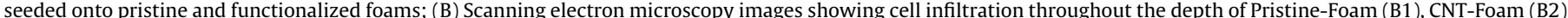

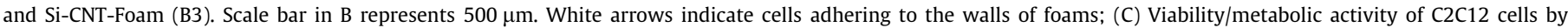

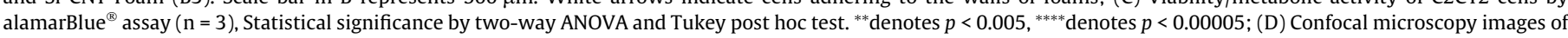

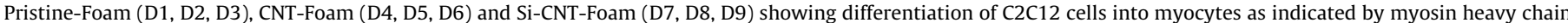

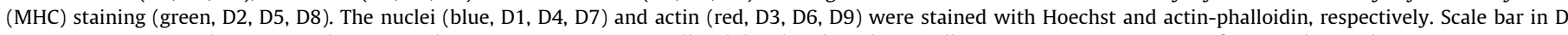

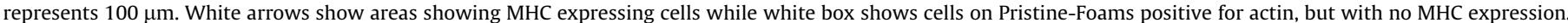

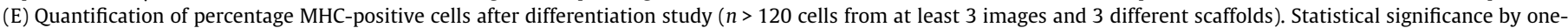
way ANOVA and Tukey post hoc test. ${ }^{* *}$ denotes $p<0.005$, ${ }^{* * *}$ denotes $p<0.0005$. 
were not statistically significant on any given day of culture, suggesting that CNT/Si-CNT carpets immobilized onto carbon foams did not significantly affect cell viability and growth. Thus, CNT/Si-CNT-Foams are found to be cytocompatible. Interestingly, we observed decrease in the metabolic activity of cells on day 14 in all three scaffolds. This can be attributed to two possible phenomena. First, we observed that confluent cell sheets peeled off from the CNT/Si-CNT-Foam surfaces as shown in representative SEM images for CNT/Si-CNT-Foam scaffolds (Supplementary Fig. 4, white arrows). SEM images also revealed comparatively smooth surfaces of CNT/Si-CNT Foam scaffolds after detachment of cell-sheets, similar to smooth surfaces observed in PristineFoams (Fig. 1B2 and Supplementary Fig. 4, denoted by asterisks).
These SEM images suggested that cell sheets peeled off along with the underlying $\mathrm{CNT} / \mathrm{Si}-\mathrm{CNT}$ carpets. We have previously reported the peeling off of CNT carpets as an entire layer from the foam surface [25]. This was attributed to the much stronger interaction between CNTs and underlying silica nanolayer than that between the graphitic layers in the underlying foam. SEM images in our study supports this conclusion and indicate that the cell sheets and $\mathrm{CNT} / \mathrm{Si}$-CNT carpets remained together during their detachment, further suggesting strong interaction of cells with underlying CNT/Si-CNT carpets. Such strong interaction between the myoblasts and CNT carpets may be beneficial in two ways. First, the CNT carpets can provide a mechanical support to the native myoblasts until they get anchored, oriented and differentiated into

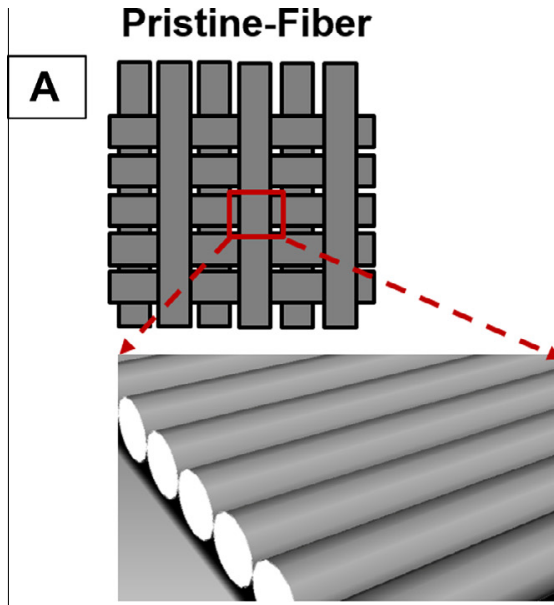

B
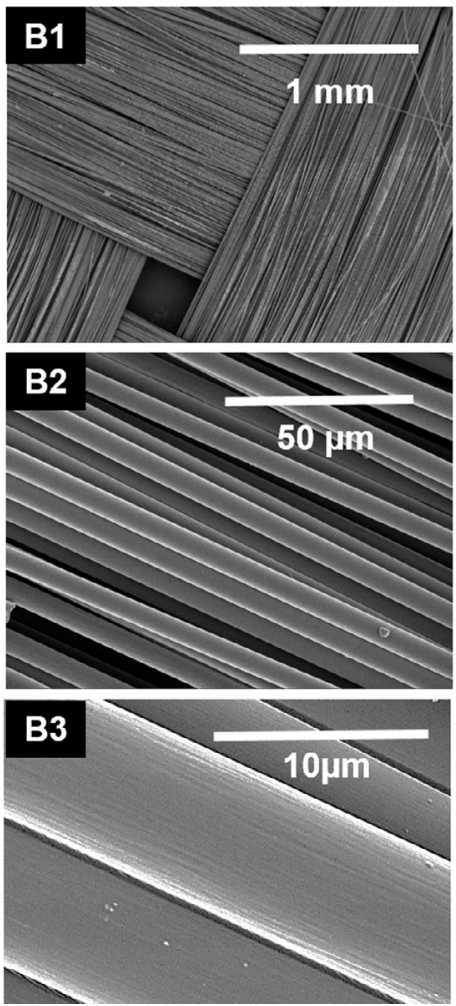

\section{CNT-Fiber}
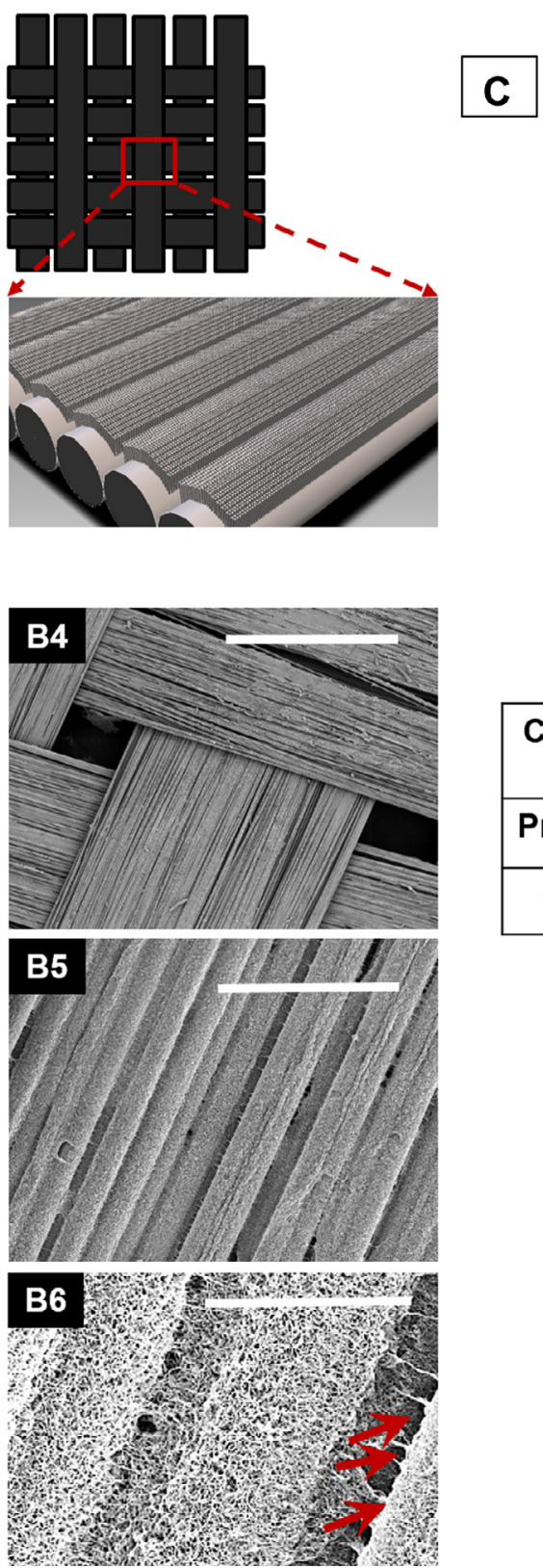
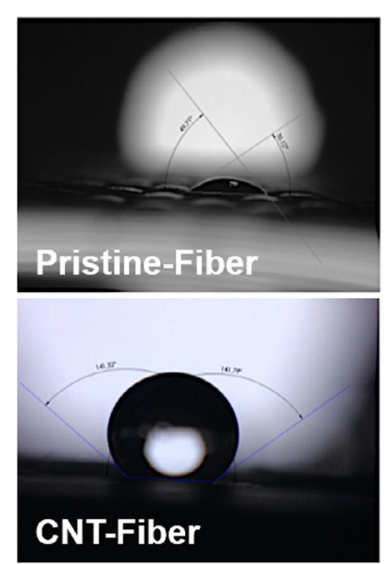

Table 3

\begin{tabular}{|c|c|}
\hline Composition & $\begin{array}{c}\text { Contact } \\
\text { angle }\left({ }^{\circ}\right)\end{array}$ \\
\hline Pristine-Fiber & 39.5 \\
\hline CNT-Fiber & 141.3 \\
\hline
\end{tabular}

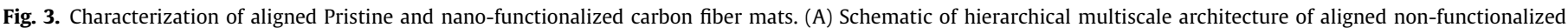

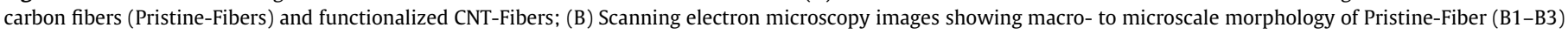

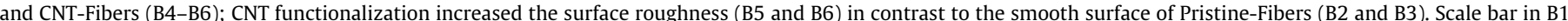

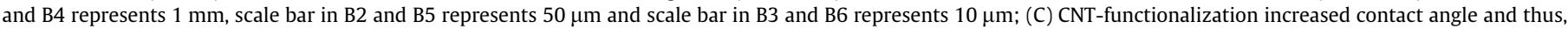
decreased wettability of the CNT-Fiber scaffolds as indicated in Table 3. 
functional multinucleated myotubes. Second, this may also prevent disaggregation/detachment of individual CNTs and their uptake by the cells, thus obviating the cytotoxicity of CNTs.

The decrease in the metabolic activity at day 14 may also be due to the differentiation of myoblast cells into multinucleated myotubes [39]. Thus, we investigated the role of CNT and Si-CNT functionalization on myoblast differentiation. C2C12 cells were cultured in growth media for 3 days, followed by 18 days in differentiation media. The cells were then fixed and stained with Hoechst (nucleus, blue), myosin heavy chain (MHC, green) and phalloidin (actin, red). Pristine-Foam scaffolds displayed attachment and spreading of $\mathrm{C} 2 \mathrm{C} 12$ cells on the foam walls as shown by nuclear and cytoskeletal actin staining (Fig. 2D1 and D3, arrows). However, very few MHC-positive cells were observed on Pristine-Foam (Fig. 2D2, white box), indicating their limited ability to differentiate into MHC-positive myocytes. On the contrary, CNTFoam (Fig. 2D4-D6) and Si-CNT-Foam (Fig. 2D7-D9) displayed presence of much higher number of MHC-positive cells, indicating that $\mathrm{CNT} / \mathrm{Si}$-CNT functionalization promoted differentiation of myoblasts into myocytes. Image analysis confirmed these results revealing significantly higher percentage of MHC-positive cells (about 90\%) in CNT-Foam and Si-CNT-Foam compared to Pristine-Foam (only 30\%, Fig. 2E). Such enhanced differentiation may be due to the nano-roughness provided by CNT/Si-CNT. This may also be due to enhanced cell-cell communication as myoblasts need electrically conductive surface for the development of functional muscle tissue and conductive CNT nanostructures may enhance transmission of such signals as suggested by others [40], although we do not have direct evidence in our current study. It should be noted that although Si coating on CNT-Foams restored their contact angle from $160^{\circ}$ to $66^{\circ}$ (similar to Pristine-Foams), altering the surface wettability did not inhibit the ability of Si-CNT-Foams to induce myoblast differentiation. On the contrary, both CNT/Si-CNT-Foam exhibited comparable percentage of MHC-positive cells despite significant differences in their contact angles (Fig. 2D5 vs. 2D8 and 2E). This suggests that nanoroughness, which is a common feature of both CNT-Foam and Si-CNT-Foam rather than wettability plays the more important role in controlling myoblast differentiation into MHC-positive cells. Similarly, Papenburg et al. reported more important role played by surface roughness on cell attachment and proliferation than surface hydrophobicity [34], which is in line with our observations in this study. Our results on the role of CNTs in promoting myoblast differentiation are in accordance with the previous reports $[28,29]$.

Although nano-functionalized foams promoted myoblast differentiation into MHC-positive myocytes, formation of multinucleated myotubes was not evident in any of the foam scaffolds. This may be due to the porous architecture of the foam scaffolds. Foams provided excellent porosity and surface area that enhanced myoblast cell proliferation and adhesion on all three scaffolds whereas CNT/Si-CNT functionalization provided additional structural cues such as nano-roughness and/or conductivity, which further aided myoblast differentiation into MHC-positive cells. However, foams

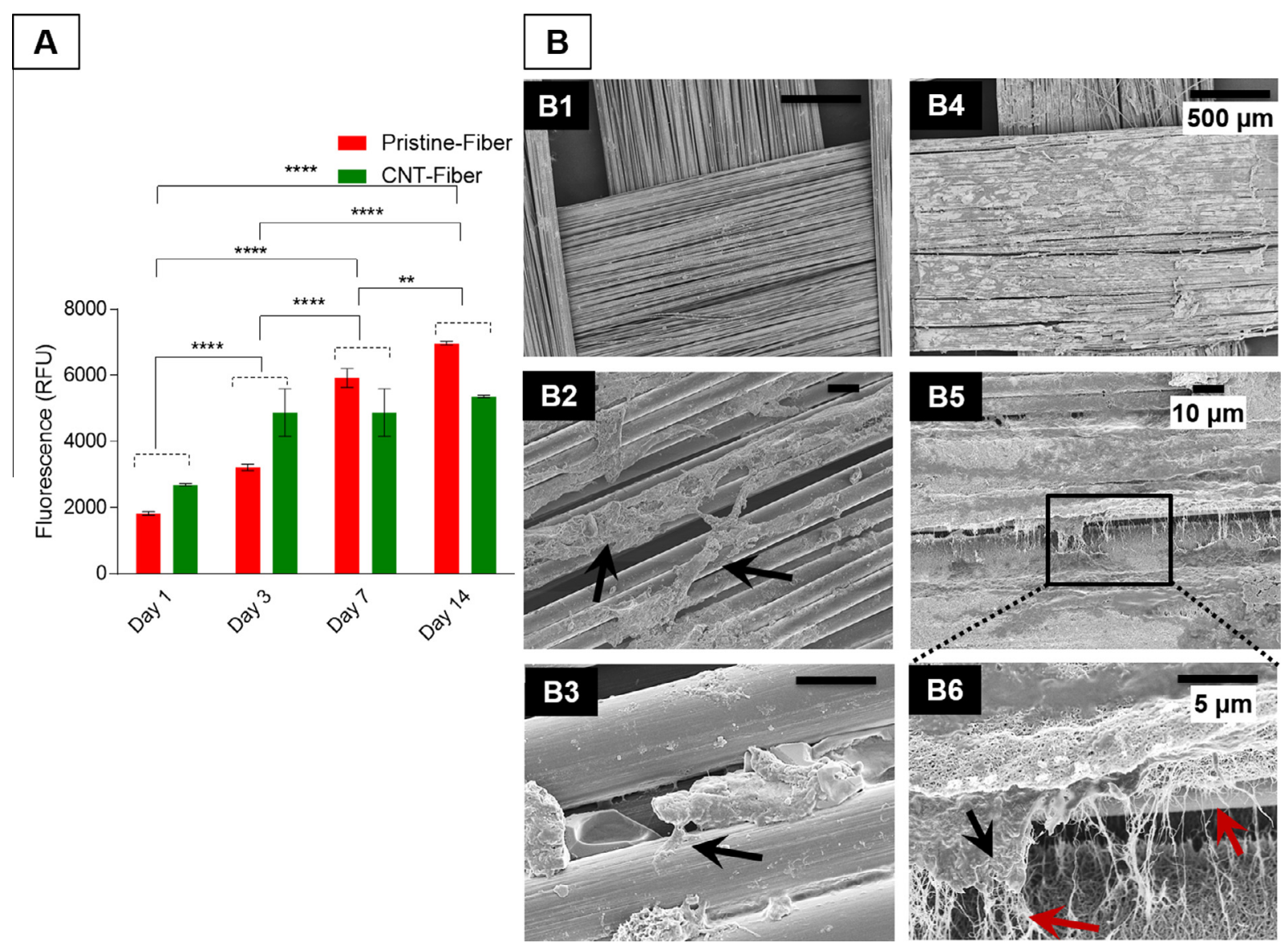

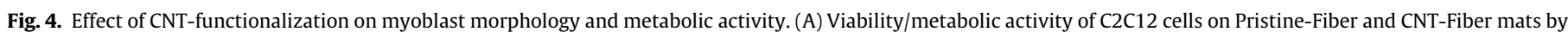

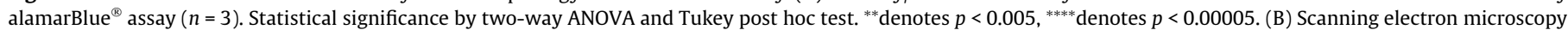

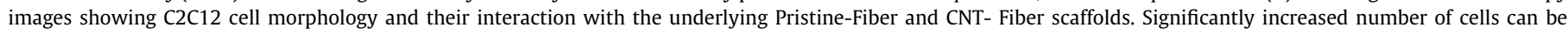

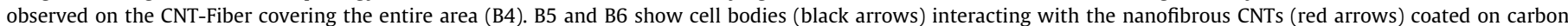

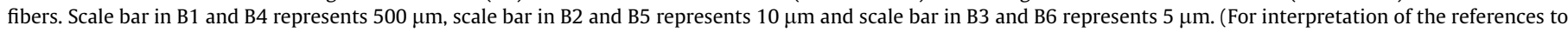
color in this figure legend, the reader is referred to the web version of this article.) 


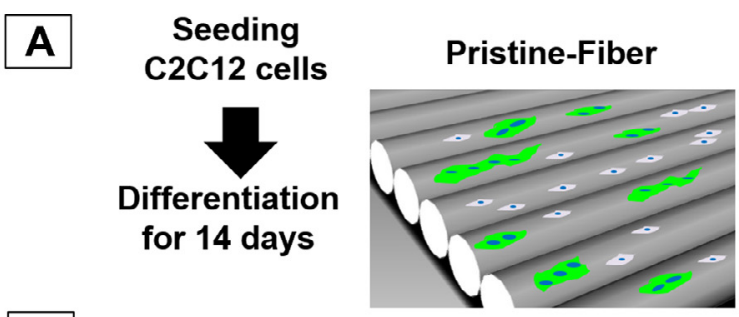

B Low magnification images
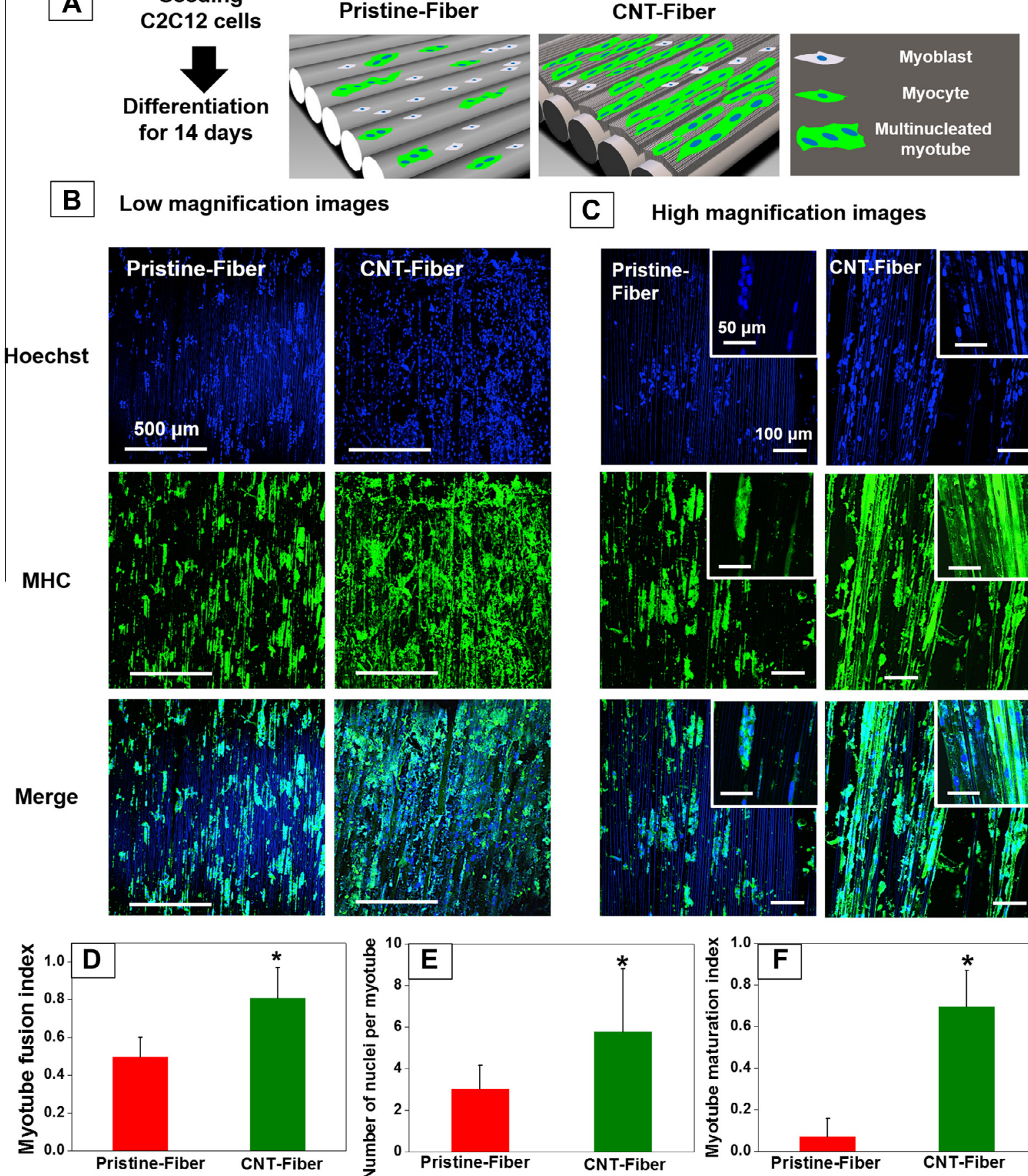

C High magnification images
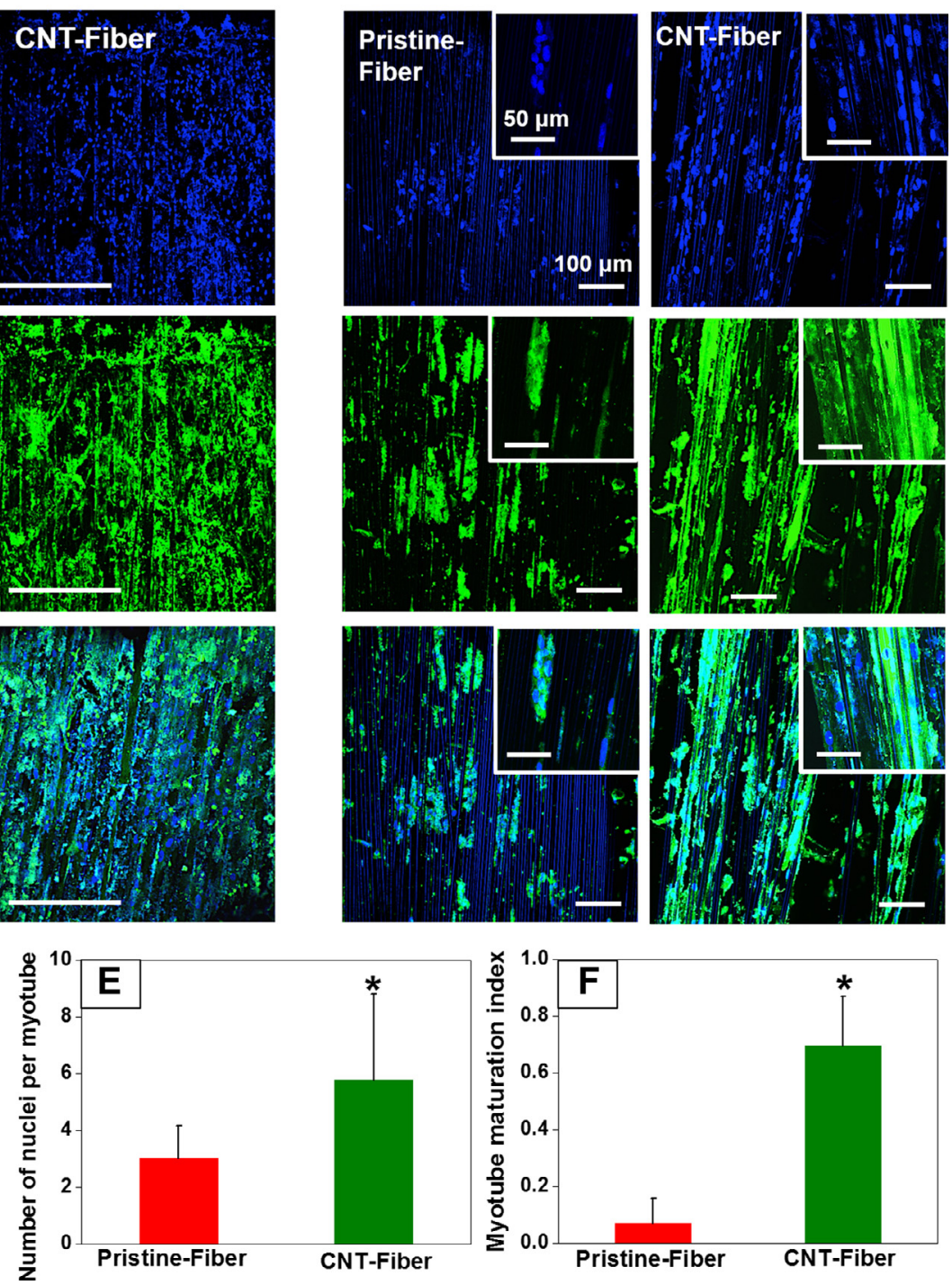

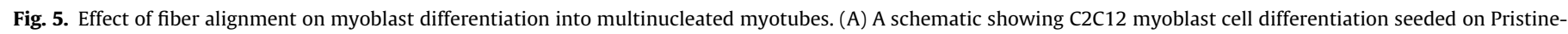

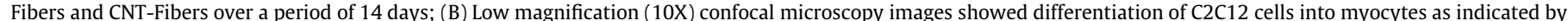

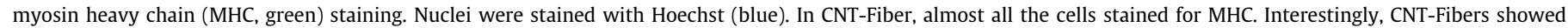

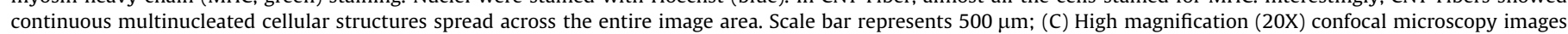

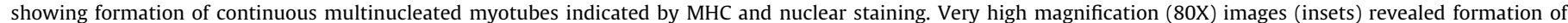

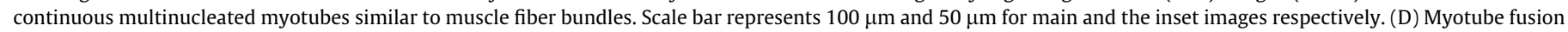

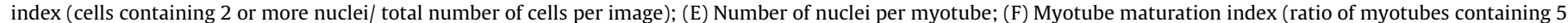

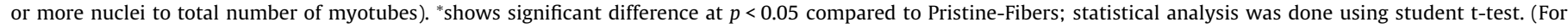
interpretation of the references to color in this figure legend, the reader is referred to the web version of this article.)

lack alignment cues that are present in native skeletal muscle tissue. In conclusion, although interconnected porous structure along with nanoscale surface functionalization led to myoblast differentiation into MHC-positive cells, it fell short of promoting fusion of these cells into multinucleated myotube-like structures.

\subsection{Characterization of aligned carbon fiber mats}

As skeletal muscle is a highly organized structure, myotube formation on surfaces without alignment cues is believed to be immature; for instance, it can form few myoblasts grown in swirled and 
unorganized pattern [41]. Such unorganized, non-aligned cellular structures may further hamper fusion and formation of multinucleated myotubes and consequently, functional regeneration of skeletal muscle. Aligned structural or topographical cues provide contact guidance, where myotubes can be formed parallel to each other leading to functional tissue development [42-45]. We, therefore, functionalized aligned woven fibrous carbon mats with strongly anchored CNT carpets using the two-step technique discussed earlier (Fig. 3A). Woven fiber mats have multiscale hierarchy with microscale fibrils forming a horizontal multilayered strip. These strips are then interwoven with a plain weave construction as shown in the schematic (Fig. 3A). As evident from the study of Si-CNT-Foam, wettability did not show any significant effect on myoblast differentiation, therefore Si functionalization was not considered in the study with carbon fiber mats. Successful CNT functionalization of fibers was confirmed by SEM imaging. Fig. 3B shows SEM micrographs of pristine carbon fibers (referred to as Pristine-Fiber hereafter) and CNT-coated carbon fibers (referred to as CNT-Fiber). After CNT grafting, the overall aligned scaffold architecture at macroscale remained unchanged while surface roughness was enhanced (Fig. 3B4-B6) compared to smooth surface observed for Pristine-Fibers (Fig. 3B1-B3). In addition, some CNTs bridging two parallel carbon fibers were observed as indicated by red arrows in Fig. 3B6. Thus, CNT grafting conferred the nano-roughness to the surface along with interconnected grooves between parallel fibers without compromising the fiber alignment. CNT grafting also increased contact angle of the scaffolds similar to CNT-Foam (Fig. 3C and Table 3).

\subsection{Effect of alignment and nano-functionalization on myoblast adhesion, proliferation and differentiation into multinucleated myotubes}

C2C12 myoblast cells were seeded on Pristine-Fiber and functionalized CNT-Fiber (Fig. 4A) to elucidate the integrated effect of fiber alignment and CNT functionalization on cellular proliferation, alignment and differentiation. Metabolic activity of seeded cells was evaluated for 14 days using alamarBlue ${ }^{\circledR}$ assay (Fig. 4A). Pristine-Fibers showed increased metabolic activity throughout 14 days while CNT-Fiber showed significantly higher metabolic activity than Pristine-Fibers until day $3(p<0.05)$, after which CNT-Fiber reached confluence and no significant change was observed thereafter. As discussed above, such reduction in metabolic activity may be attributed to stimulation of myogenic differentiation by CNT carpet. While Pristine-Fiber scaffolds showed cell adhesion/spreading and facilitated formation of multicellular structures (black arrow in Fig. 4B2), randomly orientated and less organized cell aggregates can also be observed. On the other hand, CNT-Fiber promoted formation of aligned multicellular structures completely covering the entire area of CNT-Fiber scaffold (approx. $2.5 \mathrm{~mm}$ ) as shown in Fig. 4B4 and B5. CNT-Fiber scaffolds also promoted cell-material interaction to achieve significant contact adhesion as indicated by black arrows (cell bodies) and red arrows (CNTs) (Fig. 4B6).

We further investigated the integrated influence of fiber alignment and nano-roughness of Pristine-Fiber and CNT-Fiber mats on differentiation of C2C12 myoblast cells over 14 days (Schematic in Fig. 5A). We selected earlier time point of 14 days in PristineFiber and CNT-Fiber scaffolds as most of the confluent cellular structures formed during 21 days were detached from the mats preventing any analysis of MHC-positive cells (data not shown). This suggested that aligned fibrous mats might be more efficient in promoting differentiation at earlier time point. Indeed, low magnification confocal microscopy images of cells stained for MHC (green) and nuclei (blue) showed MHC-positive cells in both Pristine-Fiber and CNT-Fiber mats in contrast to the foam scaffolds.
It is noteworthy that while Pristine-Fiber mats showed MHCpositive multinucleated cells scattered throughout the image area (Fig. 5B, left column), CNT-Fiber mats showed interconnected, multinucleated MHC-positive cells covering entire area of the image, suggesting efficient fusion of differentiated myocytes to form myotubes (Fig. 5B, right column). Higher magnification images of both Pristine and CNT-Fiber mats displayed presence of aligned myotubes parallel to the fiber direction (Fig. 5C). Interestingly, unlike Pristine-Fibers, CNT-Fibers promoted formation of continuous myotube bundles (inset of Fig. 5C), which are believed to be units of functional muscle tissue.

Image analysis further revealed significantly higher myotube fusion index in CNT-Fibers compared to Pristine-Fibers (Fig. 5D). Similarly, number of nuclei per myotube (Fig. 5E) and myotube maturation index (myotubes containing more than 5 nuclei) (Fig. 5F) were found to be significantly higher in CNT-Fiber mats compared to Pristine-Fiber mats. Immunofluorescence images of MHC-stained cells also showed striations perpendicular to the direction of myotube length in CNT-Fiber (Supplementary Fig. 5, asterisks), whereas such striations were absent in Pristine-Fiber scaffolds. Presence of such striations suggests maturity of myotubes in CNT-Fiber [46]. This observation correlates well with the 4-5 fold increase in myotube maturation index on CNT-Fiber compared to Pristine-Fiber scaffolds (Fig. 5F).

It has been reported that end-to-end fusion of myocytes or myoblasts is more feasible than their lateral fusion [47]. Alignment cues can guide appropriate cell orientation and further, promote their fusion $[44,48]$. Moreover, other studies have revealed that narrow width of aligned structural cues promoted densely packed myotubes resembling functional myotubes $[45,47]$. This supports our observations of improved myocyte fusion even in PristineFiber mats that consist of aligned fibers of about 10 micron diameter. Nano-functionalization with CNT further facilitated myocyte fusion leading to multinucleated mature myotubes.

Taken together, nano-functionalized CNT-Foams promoted myoblast differentiation into MHC-positive myocytes (Fig. 2E); however, it was not sufficient to stimulate fusion of differentiated myocytes into multinucleated myotubes (Fig. 2D6, D9). On the other hand, aligned fibrous architecture of Pristine-Fiber alone promoted myocyte differentiation as well as their fusion into multinucleated myotubes to some extent (Fig. 5C and D). More importantly, nano-functionalization interfaced with microscale aligned fibrous architecture in CNT-Fiber significantly enhanced myocyte fusion into multinucleated and mature myotubes highlighting synergy between surface nano-topography and aligned fibrous architecture (Fig. 5C-F).

\section{Conclusions}

We engineered carbon-based foam and fiber scaffolds with multiscale hierarchy conferred by nanostructured CNT carpets and microscale interconnected pores (Foam) or fibrils bundled into aligned fibers (Fiber). The Pristine-Foam scaffolds showed few MHC-positive myocytes without formation of fused and continuous myotubes. On the other hand, Pristine-Fiber mats promoted not only differentiation but also fusion of myocytes into multinucleated myotubes. This signifies that the scaffold architecture (interconnected porous vs aligned fibrous structure) is instrumental in facilitating fusion of myocytes to form multinucleated myotubes. Controlled modulation of surface wettability by nanofunctionalized CNT or Si-CNT revealed a minimal effect of wettability and a dominant role played by nano-roughness in promoting differentiation into myocytes. Nanostructured CNT carpets enhanced myoblast differentiation into myocytes in both the foam and fiber scaffolds; however, it alone was not sufficient to promote 
fusion of myocytes into multinucleated myotubes. Indeed, nanostructured CNTs combined with aligned fibrous structure facilitated formation of multinucleated myotubes and their end-to-end fusion. This was mainly due to excellent contact guidance provided by aligned fibrous architecture along with nano-roughness and conductive surface conferred by CNT carpets. In conclusion, we demonstrate that nanoscale features govern the differentiation of individual myoblasts into myocytes whereas microscale alignment cues orchestrate fusion of multiple myocytes into multinucleated myotubes underlining the importance of multiscale hierarchy in enhancing coordinated tissue regeneration.

\section{Conflict-of-interest disclosure}

The authors have no conflict-of-interest.

\section{Acknowledgement}

The authors thank Dr. Donna B. Stolz, Center for Biologic Imaging (CBI), University of Pittsburgh for access to the electron microscopy facility. SS acknowledges funding support from startup funds from the Department of Pharmaceutical Sciences at University of Pittsburgh. Mukhopadhyay group acknowledges financial support from NSF-CBET (1449582), WSU PhD program, and Ohio Board of Regents. We also thank Ultramet and Hexcel for generous supplies of carbon foam and fiber mats, respectively.

\section{Appendix A. Supplementary data}

Supplementary data associated with this article can be found, in the online version, at http://dx.doi.org/10.1016/j.actbio.2016.01. 004.

\section{References}

[1] U.S. Bureau of Labor Statistic Report. U.S. Bureau of Labor Statistic, 2011.

[2] J.T. Vilquin, Myoblast transplantation: clinical trials and perspectives. Minireview, Acta Myologica 24 (2005) 119-127.

[3] S. Sant, D.F. Coutinho, N. Sadr, R.L. Reis, A. Khademhosseini, Tissue Analogs by the Assembly of Engineered Hydrogel Blocks, Biomimetic Approaches for Biomaterials Development, Wiley-VCH Verlag GmbH \& Co. KGaAp., 2012 (pp. 471-493).

[4] P. Kerativitayanan, J.K. Carrow, A.K. Gaharwar, Nanomaterials for engineering stem cell responses, Adv. Healthcare Mater. (2015).

[5] N. Osses, E. Brandan, ECM is required for skeletal muscle differentiation independently of muscle regulatory factor expression, Am. J. Physiol. Cell Physiol. 282 (2002) C383-C384.

[6] S. Hinds, W. Bian, R.G. Dennis, N. Bursac, The role of extracellular matrix composition in structure and function of bioengineered skeletal muscle Biomaterials 32 (2011) 3575-3583.

[7] Z. Tong, S. Sant, A. Khademhosseini, X. Jia, Controlling the fibroblastic differentiation of mesenchymal stem cells via the combination of fibrous scaffolds and connective tissue growth factor, Tissue Eng. Part A 17 (2011) $2773-2785$.

[8] S. Montesano, E. Lizundia, F. D’Angelo, E. Fortunati, S. Mattioli, F. Morena, et al. Nanocomposites based on PLLA and multi walled carbon nanotubes support the myogenic differentiation of murine myoblast cell line, ISRN Tissue Eng. 2013 (2013) 8.

[9] S. Ahadian, J. Ramon-Azcon, M. Estili, X. Liang, S. Ostrovidov, H. Shiku, et al., Hybrid hydrogels containing vertically aligned carbon nanotubes with anisotropic electrical conductivity for muscle myofiber fabrication, Sci. Rep. 4 (2014) 4271.

[10] M.-C. Chen, Y.-C. Sun, Y.-H. Chen, Electrically conductive nanofibers with highly oriented structures and their potential application in skeletal muscle tissue engineering, Acta Biomater. 9 (2013) 5562-5572.

[11] Y. Tamada, Y. Ikada, Effect of preadsorbed proteins on cell adhesion to polymer surfaces, J. Colloid Interface Sci. 155 (1993) 334-339.

[12] J.E. Hall, A.C. Guyton, Guyton and Hall Textbook of Medical Physiology, Saunders Elsevier, 2011.

[13] C.M. Hwang, S. Sant, M. Masaeli, N.N. Kachouie, B. Zamanian, S.-H. Lee, et al., Fabrication of three-dimensional porous cell-laden hydrogel for tissue engineering, Biofabrication 2 (2010) 035003.
[14] A.K. Gaharwar, M. Nikkhah, S. Sant, A. Khademhosseini, Anisotropic poly (glycerol sebacate)-poly ( $\epsilon$-caprolactone) electrospun fibers promote endothelial cell guidance, Biofabrication 7 (2015) 015001.

[15] R. El-Ayoubi, N. Eliopoulos, R. Diraddo, J. Galipeau, A.-M. Yousefi, Design and fabrication of 3D porous scaffolds to facilitate cell-based gene therapy, Tissue Eng. Part A 14 (2008) 1037-1048.

[16] G.C. Engelmayr, M. Cheng, C.J. Bettinger, J.T. Borenstein, R. Langer, L.E. Freed, Accordion-like honeycombs for tissue engineering of cardiac anisotropy, Nat Mater. 7 (2008) 1003-1010.

[17] H. Park, B.L. Larson, M.D. Guillemette, S.R. Jain, C. Hua, G.C. Engelmayr Jr, et al., The significance of pore microarchitecture in a multi-layered elastomeric scaffold for contractile cardiac muscle constructs, Biomaterials 32 (2011) 1856-1864.

[18] H. Wang, X. Yuan, G. Zeng, Y. Wu, Y. Liu, Q. Jiang, et al., Three dimensional graphene based materials: Synthesis and applications from energy storage and conversion to electrochemical sensor and environmental remediation, Adv. Colloid Interface Sci. (2015).

[19] A. Xu, X. Liu, X. Gao, F. Deng, Y. Deng, S. Wei, Enhancement of osteogenesis on micro/nano-topographical carbon fiber-reinforced polyetheretherketonenanohydroxyapatite biocomposite, Mater. Sci. Eng. C Mater. Biol. Appl. 48 (2015) 592-598.

[20] D. Depan, R.D.K. Misra, The development, characterization, and cellular response of a novel electroactive nanostructured composite for electrical stimulation of neural cells, Biomater. Sci. 2 (2014) 1727-1739.

[21] N. Li, Q. Zhang, S. Gao, Q. Song, R. Huang, L. Wang, et al., Three-dimensional graphene foam as a biocompatible and conductive scaffold for neural stem cells, Sci. Rep. 3 (2013).

[22] D.B. Bennett, J.C. Hill, J. Dennison, S. O’Brien, J.L. Mantel, G.H. Isaac, et al., Metal-carbon fiber composite femoral stems in hip replacements: a randomized controlled parallel-group study with mean ten-year follow-up, J. Bone Joint Surg. Am. 96 (2014) 2062-2069.

[23] J. Chen, S. Chen, X. Zhao, L.V. Kuznetsova, S.S. Wong, I. Ojima, Functionalized single-walled carbon nanotubes as rationally designed vehicles for tumortargeted drug delivery, J. Am. Chem. Soc. 130 (2008) 16778-16785.

[24] A.K. Karumuri, L. He, S.M. Mukhopadhyay, Tuning the surface wettability of carbon nanotube carpets in multiscale hierarchical solids, Appl. Surf. Sci. 327 (2015) 122-130.

[25] S.M. Mukhopadhyay, A.K. Karumuri, I.T. Barney, Hierarchical nanostructures by nanotube grafting on porous cellular surfaces, J. Phys. D: Appl. Phys. 42 (2009) 195503.

[26] S.M. Mukhopadhyay, A.K. Karumuri, Nanotube attachment for prevention of interfacial delamination, J. Phys. D: Appl. Phys. 43 (2010) 365301.

[27] S. Mukundan, V. Sant, S. Goenka, J. Franks, L.C. Rohan, S. Sant, Nanofibrous composite scaffolds of poly(ester amides) with tunable physicochemical and degradation properties, Eur. Polym. J. 68 (2015) 21-35.

[28] K. McKeon-Fischer, D. Flagg, J. Freeman, Coaxial electrospun poly ( $\varepsilon-$ caprolactone), multiwalled carbon nanotubes, and polyacrylic acid/polyvinyl alcohol scaffold for skeletal muscle tissue engineering, J. Biomed. Mater. Res. A 99 (2011) 493-499.

[29] G.M. Spinks, V. Mottaghitalab, M. Bahrami-Samani, P.G. Whitten, G.G. Wallace, Carbon-nanotube-reinforced polyaniline fibers for high-strength artificial muscles, Adv. Mater. 18 (2006) 637-640.

[30] S.R. Shin, S.M. Jung, M. Zalabany, K. Kim, P. Zorlutuna, Sb Kim, et al., Carbonnanotube-embedded hydrogel sheets for engineering cardiac constructs and bioactuators, ACS Nano 7 (2013) 2369-2380.

[31] K.M. Ferlin, D.S. Kaplan, J.P. Fisher, Characterization of the adhesive interactions between cells and biomaterials, Micro and Nanotechnologies in Engineering Stem Cells and Tissues, John Wiley \& Sons, Inc., 2013 (pp. 159-182).

[32] J.A. Chinn, Biomaterials: protein-surface interactions, in: J.D. Bronzino (Ed.), Biomedical Engineering Handbook, CRC Press, Inc./IEEE Press, 1995, pp. 15971608.

[33] J.M. Rice, J.A. Hunt, J.A. Gallagher, P. Hanarp, D.S. Sutherland, J. Gold, Quantitative assessment of the response of primary derived human osteoblasts and macrophages to a range of nanotopography surfaces in a single culture model in vitro, Biomaterials 24 (2003) 4799-4818.

[34] B.J. Papenburg, E.D. Rodrigues, M. Wessling, D. Stamatialis, Insights into the role of material surface topography and wettability on cell-material interactions, Soft Matter 6 (2010) 4377-4388.

[35] A.K. Gaharwar, S.M. Mihaila, A. Swami, A. Patel, S. Sant, R.L. Reis, et al., Bioactive silicate nanoplatelets for osteogenic differentiation of human mesenchymal stem cells, Adv Mater. 25 (2013) 3329-3336.

[36] L. Lacerda, A. Bianco, M. Prato, K. Kostarelos, Carbon nanotubes as nanomedicines: from toxicology to pharmacology, Adv. Drug Deliv. Rev. 58 (2006) 1460-1470.

[37] X. Zhang, W. Hu, J. Li, L. Tao, Y. Wei, A comparative study of cellular uptake and cytotoxicity of multi-walled carbon nanotubes, graphene oxide, and nanodiamond, Toxicol Res. 1 (2012) 62-68.

[38] S. Sant, D. Iyer, A.K. Gaharwar, A. Patel, A. Khademhosseini, Effect of biodegradation and de novo matrix synthesis on the mechanical properties of valvular interstitial cell-seeded polyglycerol sebacate-polycaprolactone scaffolds, Acta Biomater. 9 (2013) 5963-5973.

[39] S. Sirivisoot, B.S. Harrison, Skeletal myotube formation enhanced by electrospun polyurethane carbon nanotube scaffolds, Int. J. Nanomed. 6 (2011) 2483-2497.

[40] J. Meng, L. Song, J. Meng, H. Kong, G. Zhu, C. Wang, et al., Using single-walled carbon nanotubes nonwoven films as scaffolds to enhance long-term cell proliferation in vitro, J. Biomed. Mater. Res. A 79 (2006) 298-306. 
[41] S.A. Riboldi, M. Sampaolesi, P. Neuenschwander, G. Cossu, S. Mantero, Electrospun degradable polyesterurethane membranes: potential scaffolds for skeletal muscle tissue engineering, Biomaterials 26 (2005) 46064615.

[42] J.S. Choi, S.J. Lee, G.J. Christ, A. Atala, J. Yoo, The influence of electrospun aligned poly ( $\varepsilon$-caprolactone)/collagen nanofiber meshes on the formation of self-aligned skeletal muscle myotubes, Biomaterials 29 (2008) 2899-2906.

[43] M.T. Lam, Y.-C. Huang, R.K. Birla, S. Takayama, Microfeature guided skeleta muscle tissue engineering for highly organized 3-dimensional free-standing constructs, Biomaterials 30 (2009) 1150-1155.

[44] P. Clark, D. Coles, M. Peckham, Preferential adhesion to and survival on patterned laminin organizes myogenesis in vitro, Exp. Cell Res. 230 (1997) $275-283$.
[45] D.J.R. Evans, S. Britland, P.M. Wigmore, Differential response of fetal and neonatal myoblasts to topographical guidance cues in vitro, Dev. Genes. Evol. 209 (1999) 438-442.

[46] P.F. van der Ven, G. Schaart, P.H. Jap, R.C. Sengers, A.M. Stadhouders, F.C Ramaekers, Differentiation of human skeletal muscle cells in culture: maturation as indicated by titin and desmin striation, Cell Tissue Res. 270 (1992) 189-198.

[47] R.M. Duffy, A.W. Feinberg, Engineered skeletal muscle tissue for soft robotics: fabrication strategies, current applications, and future challenges, Wiley Interdiscip. Rev. Nanomed Nanobiotechnol. 6 (2014) 178-195.

[48] P. Clark, G.A. Dunn, A. Knibbs, M. Peckham, Alignment of myoblasts on ultrafine gratings inhibits fusion in vitro, Int. J. Biochem. Cell Biol. 34 (2002) 816-825. 